\title{
Uso del taping en el manejo clínico de sujetos con pinzamiento sub-acromial: revisión sistemática
}

\author{
R. A. Aguilera Eguía $a^{1,2,3,4}$ E. O. Zafra Santos ${ }^{2,3}$, F. A. Araya Quintanilla ${ }^{4}$, L. E. Gómez Carreño ${ }^{4}$
} J. X. Soto Aliaga 4 , A. Vargas Vargas ${ }^{4}$, C. J. Cofré Bolados ${ }^{1,2,3}$, A. R. Espinoza Salinas ${ }^{2,3,4}$, G. A. Pavez von Martens $s^{2,3,4}$, T. Aguilera Eguía y M. Di Santo

${ }^{1}$ Universidad Santiago de Chile, Facultad Ciencias Médicas. ${ }^{2}$ Universidad Santo Tomas. Escuela de Kinesiología. Sede Santiago. ${ }^{3}$ Laboratorio Fisiología del Ejercicio. Escuela de Kinesiología. Universidad Santo Tomás. Santiago, Chile. ${ }^{4}$ Kinesiólogo. ${ }^{5}$ Universidad Alberto Hurtado. Facultad de Educación. Santiago de Chile. ${ }^{6}$ Instituto del Profesorado en Educación Física. Córdoba, Argentina

Aguilera Eguía RA, Zafra Santos EO, Araya Quintanilla FA, Gómez Carreño LE, Soto Aliaga JX, Vargas Vargas A, Cofré Bolados CJ, Espinoza Salinas AR, Pavez von Martens GA, Aguilera Eguía T, Di Santo M. Uso del taping en el manejo clínico de sujetos con pinzamiento sub-acromial: revisión sistemática. Rev Soc Esp Dolor 2014; 21(1): 39-49.

\begin{abstract}
The sub-acromial impingement (SAI) is the most common musculoskeletal disorder of shoulder pathologies. In recent times, the taping has become a relatively new and quite common indication in recent years. There will be a synthesis of evidence through a systematic review (SR) of randomized trials that had compared the use of taping for the management of these patients. The aim of this review is to determine the effect of taping on pain in subjects with sub-acromial pinch. The research strategy included randomized clinical trials (RCTs) databases were used: Medline, Cinahl, PEDro, Central, LILACS. In addition, we searched electronic journals, conference proceedings, randomized trials registry and contacted experts in the field. The results were two articles that met our eligibility criteria. We conclude that there is low quality evidence suggesting that the application of taping, would be effective to improve functionality and reduce pain at rest and during movements up to 1 week after application, in subjects with sub-acromial pinch compared to physical therapy program.
\end{abstract}

Key words: Sub-acromial impingement. Shoulder impingement syndrome. Rotator cuff. Adhesive tape. Orthotic tape. Taping. Taping shoulder. Kinesiotape.

\section{RESUMEN}

El pinzamiento sub-acromial (SPS) es el trastorno músculo-esquelético más frecuente de las patologías de hombro. El taping se ha transformado en una indicación relativamente nueva y bastante común en los últimos años. Se realizará una sintesis de la evidencia a través de una revisión sistemática (RS) de ensayos clínicos aleatorizados que hayan comparado el uso del taping para el manejo de estos pacientes. El objetivo de esta revisión es determinar el efecto del taping sobre el dolor en sujetos con pellizcamiento sub-acromial. La estrategia de búsqueda incluyó ensayos clínicos aleatorizados (ECA); las bases de datos usadas fueron: Medline, Cinahl, PEDro, Central, LILACS. Además, se realizó una búsqueda en revistas electrónicas, actas de congresos, Registro de Estudios Clínicos Aleatorizados y se contactó con expertos en el tema. Los resultados obtenidos fueron de dos artículos que cumplían con nuestros criterios de elegibilidad. Se concluye que existe evidencia de baja calidad que sugiere que la aplicación de taping sería efectiva para mejorar la funcionalidad, reducir el dolor en reposo y durante los movimientos hasta en una semana posterior a su aplicación, en sujetos que presentan pellizcamiento sub-acromial en comparación a un programa de terapia física.

Palabras clave: Pellizcamiento sub-acromial. Síndrome de pellizcamiento sub-acromial de hombro. Manguito rotador. Vendaje adhesivo. Vendaje ortésico. Taping. Taping de hombro. Kinesiotaping.

\section{DESCRIPCIÓN DEL SÍNDROME}

Las patologías de hombro son consideradas como uno de los trastornos músculo-esqueléticos (MEQ) más comunes en la población (1-3), siendo el síndrome de pinzamiento sub-acromial (SPS) el diagnóstico más frecuente 
Rev. Soc. Esp. del Dolor, Vol. 21, N. ${ }^{o}$ 1, Enero-Febrero 2014

alcanzando un $65 \%$ de motivo de consulta (4-7). Se estima que el $40 \%$ de las personas lo presenta en el algún momento de la vida, teniendo una prevalencia en personas mayores de 70 años de un $21 \%$ en España (11,2 por mil pacientes en un año).

Neer $(8,9)$ fue el primero en utilizar este término quien lo define como: "una compresión y/o fricción entre el tendón del músculo supra-espinoso y los tejidos circundantes del espacio sub-acromial" (10-14). Las causas del SPS se pueden atribuir a diversos factores, entre ellos: la inflamación de la estructuras contenidas en el espacio sub-acromial (10,11,14-16), el estrechamiento de la salida del músculo supra-espinoso, ya sea por hipertrofia o contractura de este músculo $(9,17-21)$, otros factores anatómicos tales como: un acromion en forma de gancho $(8,13,22,23)$, presencia de osteofitos en la articulación acromio-clavicular $(8,13,22,23)$, o de tipo más funcional, por la inestabilidad dinámica de la escápula $(24,25)$ que se asocia a la cinemática alterada del mismo complejo (14,26-30).

En cuanto a la clínica de los pacientes que presentan SPS es bastante característica. La mayoría de los sujetos refieren dolor de hombro al momento de elevar la extremidad superando los $90^{\circ}$, se observa disminución del rango activo de hombro $(31,32)$, pérdida de fuerza muscular $(31,32)$, y alteración en las actividades funcionales de la extremidad superior (EESS) $(31,32)$.

\section{DESCRIPCIÓN DE LA INTERVENCIÓN}

Existen distintos tratamientos enfocados a disminuir la sintomatología e incrementar la funcionalidad en pacientes que presentan SPS, en los cuales se encuentra el tape o taping $(33,34)$.

Este vendaje es un método relativamente nuevo y bastante común en los últimos años (23). Fue desarrollado por Kenzo Kase en $1973(23,36,37)$ y es tipo de cinta fija que tiene el mismo peso $(23,37)$ y grosor de la piel $(23,41)$. Su elasticidad permite un alargamiento de 130-140\% en su estado de reposo $(23,34-37)$. Esta cinta se adhiere a la piel siendo ubicada según la técnica a aplicar; sin embargo, las más utilizadas son las pertenecientes a los siguientes tipos: tipo muscular, que activa o limita la función de un músculo; tipo estructural, que corrige una postura anormal adoptada por algún deterioro; y el tipo circulatorio, que ayuda a la cicatrización y drenaje en las zonas donde es ubicado.

Diversos efectos son atribuidos al uso del taping, dentro de los cuales se encuentran: disminución del dolor $(34,35,38-52)$, incremento de la funcionalidad $(35,38,40,41,47-50)$ y propiocepción $(40,43,44,53-58)$. También se ha reportado un aumento del rango de movimiento $(35,38,48-50,59)$, mejoras en el drenaje linfático (23,35,60-62), movilidad escapular (63), soporte de músculos débiles $(35,63)$ y corrección de desalineamiento articular $(35,43,64)$. Por esto es que esta intervención es ampliamente utilizada en el campo de la rehabilitación como herramienta útil, tanto como un medio de tratamiento, como en la prevención de las lesiones deportivas $(43,64,65)$.

Actualmente, el uso del taping se está llevando a cabo como terapia paralela a la brindada por el fisioterapeuta, principalmente orientada a mantener la evolución alcanzada durante las intervenciones. Sin embargo, varios autores mencionan que la función de soporte del tape se pierde dentro de un tiempo relativamente corto después de su aplicación (68-70).

\section{CÓMO PODRÍA FUNCIONAR LA INTERVENCIÓN}

El uso del tape en el contexto clínico ha aumentado en el último tiempo (23) y los fundamentos para su aplicación se basan en los propuestos por Kase y cols. $(35,36)$ los cuales consisten en: producir una corrección en el desbalance muscular, ya sea en musculatura sobre-activada y/o debilitada (35), mejorar la circulación linfática mediante la eliminación de fluido debajo de la piel, eliminando estos desechos (3), mejorar la circulación estimulando la irrigación en el punto donde se ubica la cinta y disminuir el dolor a través de la teoría del Gate Control.

Murray y cols. (49) adicionaron otro mecanismo, incremento en la propiocepción, el cual se podría obtener a través de la estimulación incrementada de los mecano-receptores cutáneos; esto se debe a que al adherir la cinta a la piel se estimulan propioceptores locales que ayudarían a entregar inputs a la articulación o la zona donde se encuentra el vendaje.

En la práctica clínica se han obtenido efectos beneficiosos, pero desde el punto de vista de la evidencia científica no existen estudios de revisión concluyentes que puedan demostrar los efectos otorgados al taping ni un claro consenso en aspectos esenciales de la técnica como la duración que se debe mantener, el vendaje o la longitud elástica que alcanza.

\section{POR QUÉ ES IMPORTANTE REALIZAR ESTA REVISIÓN}

Actualmente existe una revisión sistemática que analiza los efectos del taping sobre la propiocepción, reclutamiento muscular, dolor y recuperación funcional en sujetos que presentan pellizcamiento sub-acromial.

El trabajo fue realizado por Cooping y cols. (71) siendo una RS que presenta una estrategia de búsqueda hasta el año 2004, el cual presenta limitaciones metodológicas como: restricción de lenguaje, la búsqueda no fue realizada por 2 investigadores de forma independiente, no realizaron 
búsquedas manuales en revistas electrónicas, no siguieron referencias de artículos relevantes, no revisaron registros de estudios, no consultaron actas de congresos, no contactaron a expertos en el tema, y no realizaron análisis de sensibilidad ni de subgrupos. También es relevante mencionar que la RS no presenta un protocolo previo y a su vez no reporta los niveles de evidencia y grados de recomendación con la metodología GRADE. Por tal motivo creemos que es necesario actualizar la RS.

De lo anteriormente expuesto nace nuestra pregunta de investigación: en pacientes con pellizcamiento sub-acromial, ¿el uso del taping solo o en conjunto con otras intervenciones terapéuticas disminuye el dolor?

\section{OBJETIVOS}

Determinar el efecto del taping sobre el dolor en sujetos con pellizcamiento sub-acromial.

\section{MÉTODOS}

Criterios para considerar los estudios en esta revisión

\section{Tipo de estudios}

Sólo incluiremos ensayos clínicos aleatorizados.

\section{Tipos de participantes}

\section{Criterios de inclusión}

Sólo estudios clínicos que involucren a sujetos con diagnóstico de pellizcamiento sub-acromial.

Deben presentar dolor en la zona antero-lateral del hombro al realizar una elevación o abducción de hombro por sobre los $90^{\circ} \mathrm{y} / \mathrm{o}$ a la palpación a nivel del acromion. Estos síntomas se evalúan con uno o más test específicos para el SPS (como por ejemplo test de Neer y test de Hawkins y Kennedy).

\section{Criterio de exclusión}

No incluiremos comparaciones con cirugía.

\section{Tipos de intervenciones}

Estudios clínicos que involucren todas las formas de taping aplicadas en la región del hombro.
El taping se puede utilizar solo o en conjunto con otras intervenciones.

Las intervenciones control pueden ser todos los tipos de tratamientos conservadores (vendas placebo, ultrasonido terapéutico, láser, masaje, electroterapia, tratamiento tópico, manipulación, inyección de corticoides).

Se incluirán todos los comparadores no quirúrgicos.

En la presente revisión investigaremos las siguientes comparaciones de tratamiento:

1. Taping solo versus ningún tratamiento.

2. Taping solo versus placebo.

3. Taping solo versus otros tratamientos.

\section{Outcome primarios}

Estudios que hayan utilizado una o más de las siguientes medidas de resultado:

- Funcionalidad (DASH).

- Dolor (medidos por escala visual análoga, escala numérica) en reposo o durante las actividades.

\section{Outcome secundarios}

- Cualquier efecto adverso.

\section{MÉTODO DE BÚSQUEDA PARA LA IDENTIFICACIÓN DE ESTUDIOS}

Se realizó una búsqueda en el registro especializado Cochrane del Grupo Músculo-esquelético (hasta enero 2013), Registro Cochrane de Ensayos Controlados (CENTRAL) (hasta enero 2013) y MEDLINE (hasta enero 2013).

Estas búsquedas se llevaron a cabo utilizando el algoritmo recomendado por la Colaboración Cochrane (72).

No hubo restricción de idioma; los estudios que cumplían con nuestros criterios de inclusión fueron seleccionados y se analizó su calidad metodológica

La selección de los estudios, la evaluación de la calidad metodológica y la extracción de los datos fue realizada por dos autores (LG-JS) de forma independiente; en caso de existir algún desacuerdo, un tercer autor (FA) tomaba la decisión.

Se realizó una búsqueda manual desde 1986 a enero 2013 en las siguientes revistas electrónicas: Alexandria Journal of Medicine, Acute Pain, Archives of Physical Medicine and Rehabilitation, Archives of Physical Medicine and Rehabilitation, Physiotherapy, Physical Therapy in Sport, Journal of Shoulder and Elbow Surgery, Journal of Pain and Symptom Management, Journal of Hand Therapy, Journal of Electromyography and Kinesiology, Hong Kong 
Physiotherapy Journal, Clinical Biomechanics, Best Practice \& Research Clinical Rheumatology, Physical Therapy Reviews, Federación Española de Medicina del Deporte, Journal of Applied Clinical Medical Physics, Journal of Chiropractic Education, Journal of Chiropractic Medicine, The Journal of Manual \& Manipulative Therapy, Journal of Orthopaedic Surgery and Research, The Journal of Sports Science and Medicine, The Journal of the Canadian Chiropractic Association, New Zealand Society of Physiotherapists Osteopathic Medicine and Primary Care, American Physical Therapy Association, Revista Educación en Ciencias de la Salud, Revista Educación de Medicina y Ciencia de la Actividad Física y del Deporte, Revista Internacional de Ciencias del Deporte, Sports Medicine, Rehabilitation, Therapy \& Technology, Sportscience, Revista Cubana de Ortopedia y Traumatología, Revista Mexicana de Medicina Física y Rehabilitación, Revista Mexicana de Ortopedia Pediátrica, Revista Universitaria en Ciencias de la Salud, Asociación Argentina de Ortopedia y Traumatología, Colegio de kinesiólogos de la Provincia de Buenos Aires, Revista Brasileira de Ortopedia, Revista Chilena de Ortopedia y Traumatología, Revista Colombiana de Ortopedia y Traumatología, Revista Venezolana de Cirugía y Traumatología, Revista Boliviana de Ortopedia y Traumatología, Revista Latinoamericana de Ortopedia.

Realizamos seguimiento de las referencias de los artículos relevantes y consultamos actas de los siguientes congresos: Congreso de la Sociedad Española de Cirugía Ortopédica y Traumatología (SECOT, 2012), Acta Ortopédica de Bélgica (1991-2012), Congreso Sociedad de Traumatología y Cirugía Ortopédica de la Comunidad Autónoma de Valencia (SOTOCAV, 2006-2013), Congreso Argentino de Ortopedia y Traumatología (2011).

Se realizó una búsqueda de protocolos de estudios clínicos aleatorizados en los siguientes registros: GlaxoSmithKline Clinical Trials Registry (GSK), Lilly Trials Eli Lilly and Company, registro de ensayos clínicos (CIRCARE) y la Plataforma de Registros Internacionales de Ensayos Clínicos (ICTRP) de la Organización Mundial de la Salud.

Además buscamos en Google Scholar, OVID (1950 a 2013), CINHAL (1982 a 2013), PEDro (1985-2013), LILACS (1986-2013).

Para MEDLINE utilizamos la siguiente estrategia de búsqueda que incluye una combinación de vocabulario controlado y términos de texto libre:

1. Sub-acromial impingement

2. Shoulder impingement syndrome

3. Rotator cuff

4. ((\#1) OR \#2) OR \#3

5. Adhesive tape

6. Athletic tape

7. Orthotic tape

8. Taping
9. Taping shoulder

10. Kinesiotape

11. ((( (\#5) OR \#6) OR \#7) OR \#8) OR \#9) OR \#12

12. (\#4) AND \#11

13. Randomized clinical trial

14. Randomized controlled trial

15. Randomized

16. ((\#13) OR \#14) OR \#15

17. Humans

18. Animals

19. (\#17) NOT \#18

20. ((\#11) AND \#16) AND \#19

\section{RIESGO DE SESGO Y VALORACIÓN DE LA CALIDAD METODOLÓGICA}

Se evaluó el riesgo de sesgo para cada estudio incluido, evaluando el rigor del proceso de aleatorización, ocultamiento de la asignación, el cegamiento, datos de resultados incompletos y reporte de seguimiento de los resultados como recomienda el Manual de la Colaboración Cochrane (73).

Los estudios incluidos en nuestra revisión fueron evaluados cualitativamente y los resultados se expresan en la Tabla I (Análisis del riesgo de sesgo) y la figura 1 (Resumen del análisis del riesgo de sesgo), especificando si cumple el criterio (color verde: bajo riesgo de sesgo), no lo cumple (rojo: alto riesgo de sesgo) o es poco claro (amarillo: riesgo incierto/poco claro/falta información para dar una sentencia).

TABLA I. RIESGO DE SESGO DE ARTÍCULOS INCLUIDOS

\begin{tabular}{|c|c|c|}
\hline $\begin{array}{c}\text { Thelen } \\
2008\end{array}$ & $\begin{array}{l}\text { Kaya } \\
2011\end{array}$ & \\
\hline+ & - & Generación de la secuencia aleatoria \\
\hline$?$ & - & Ocultamiento de la asignación \\
\hline+ & + & $\begin{array}{l}\text { Cegamiento de los participantes y del } \\
\text { personal (Dolor) }\end{array}$ \\
\hline+ & + & $\begin{array}{l}\text { Cegamiento de los participantes y del } \\
\text { personal (Funcionalidad) }\end{array}$ \\
\hline+ & $?$ & $\begin{array}{l}\text { Cegamiento de la evaluación de los } \\
\text { resultados (Dolor) }\end{array}$ \\
\hline+ & $?$ & $\begin{array}{l}\text { Cegamiento de la evaluación de los } \\
\text { resultados (Funcionalidad) }\end{array}$ \\
\hline+ & + & Datos de resultados incompletos \\
\hline$?$ & $?$ & Notificación selectiva de los resultados \\
\hline+ & + & Otros sesgos \\
\hline
\end{tabular}




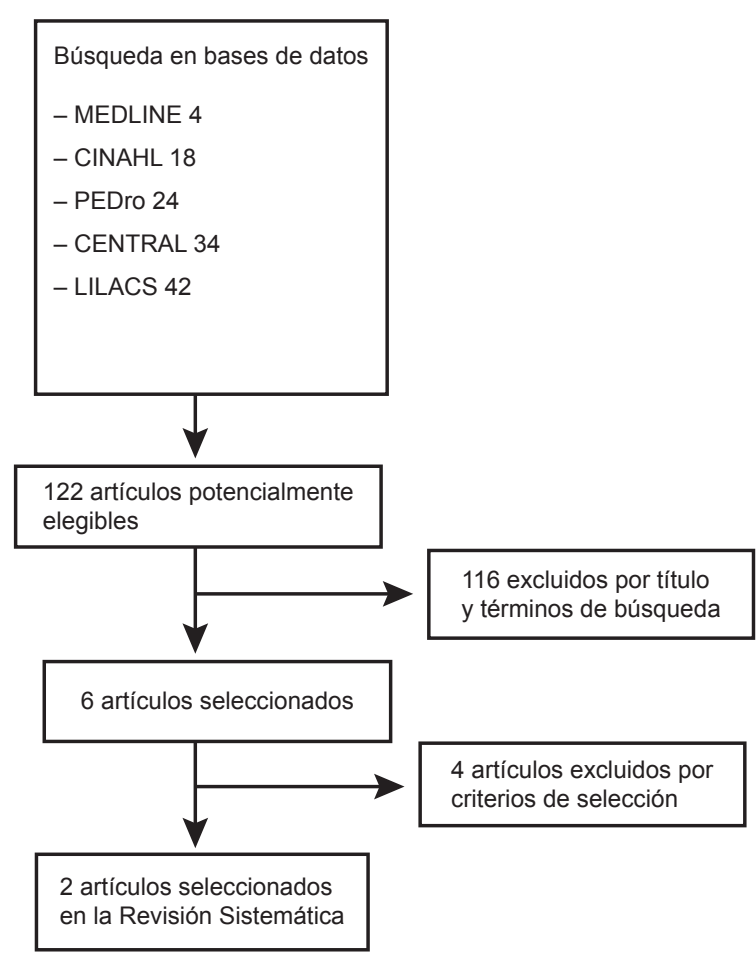

Fig. 1. Algoritmo de búsqueda.

Los siete criterios a evaluar son:

- Generación de la secuencia aleatoria.

- Ocultamiento de la secuencia de aleatorización.

- Cegamiento de los participantes y del personal.

- Cegamiento de la evaluación de los resultados.

- Datos de resultados incompletos.

- Notificación selectiva de los resultados.

- Otros sesgos.

La calidad global de la evidencia será evaluada usando la metodología GRADE (77). La calidad de la evidencia de un resultado específico se basa en los siguientes puntos:

1. Limitación en el diseño (metodológicas).

2. Inconsistencia de los resultados.

3. Evidencia indirecta.

4. Imprecisiones.

5. Sesgo de publicación.

La calidad de la evidencia se describe de la siguiente forma:

- Alta calidad: es poco probable que investigaciones adicionales cambien nuestra confianza en la estimación del efecto. Hay suficientes datos con intervalos de confianza estrechos.

- Moderada calidad: es probable que investigaciones adicionales tengan un impacto importante en la confianza de la estimación del efecto y puedan cambiar la estimación.

- Baja calidad: es muy probable que investigaciones adicionales tengan un impacto importante en la con- fianza de la estimación del efecto y es probable que cambie.

- Calidad muy baja: presenta una gran incertidumbre sobre la estimación del efecto.

- Ninguna evidencia: no hay evidencia de ECA.

\section{SÍNTESIS Y ANÁLISIS DE DATOS}

Partiendo del análisis de los datos extraídos de los artículos que cumplían los criterios de elegibilidad de nuestra revisión sistemática, y al comparar taping versus placebo y taping versus otros tratamientos, no se pudieron agrupar los datos debido a la diversidad de intervenciones incluidas en los estudios.

Para el análisis de los datos se usó el programa RevMan 5.

\section{MEDIDAS DE RESULTADO}

Las medidas de resultados más comúnmente utilizadas en los artículos seleccionados fueron: el dolor $(62,74)$, en cuanto a su medición se utilizó la escala visual análoga (EVA), que es una escala de $100 \mathrm{~mm}$ para evaluar la intensidad del dolor (62), el índice de discapacidad y dolor de hombro (SPADI) (62), que es un cuestionario de 13 ítems que consta de 2 subescalas, una de dolor (5 artículos) y discapacidad ( 8 artículos); el resultado es el promedio de las 2 subescalas que va de 0 a 100 puntos, cuanto más alto sea el resultado, mayor es el dolor y discapacidad. Otro de los resultados evaluados fue el rango de movimiento (ROM) (62) en la articulación de hombro mediante un goniómetro estándar para la flexión anterior, abducción y elevación en el plano escapular. Para medir la funcionalidad $(62,74)$, se utilizó la escala de discapacidad de brazo, hombro y mano (DASH) (74).

\section{RESULTADOS}

De acuerdo a los criterios de nuestra revisión, la búsqueda preliminar identificó 122 artículos potencialmente elegibles al aplicar los límites de búsqueda y los criterios de selección; quedaron 2 estudios, todo se detalla en el cronograma de búsqueda de la RS que se muestra en la figura 2, de los cuales ambos corresponden a ECA ( $\mathrm{n}=97$ pacientes) $(62,74)$. Todos los estudios se realizaron en pacientes con pinzamiento sub-acromial. Las comparaciones realizadas fueron las siguientes: taping versus ninguna intervención, taping versus placebo ( $\mathrm{n}=42$ pacientes) y taping versus otros tratamientos $(n=55)$, el rango de edad de los pacientes estaba entre 19 y 59 años con un promedio de 39 años. 
Generación de la secuencia aleatoria

Ocultamiento de la asignación

Cegamiento de los participantes y del personal (Dolor)

Cegamiento de los participantes y del personal (Funcionalidad)

Cegamiento de la evaluación de los resultados (Dolor)

Cegamiento de la evaluación de los resultados (Funcionalidad)

Datos de resultados incompletos

Notificación selectiva de los resultados

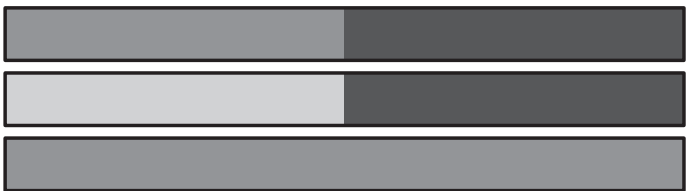

Otros sesgos
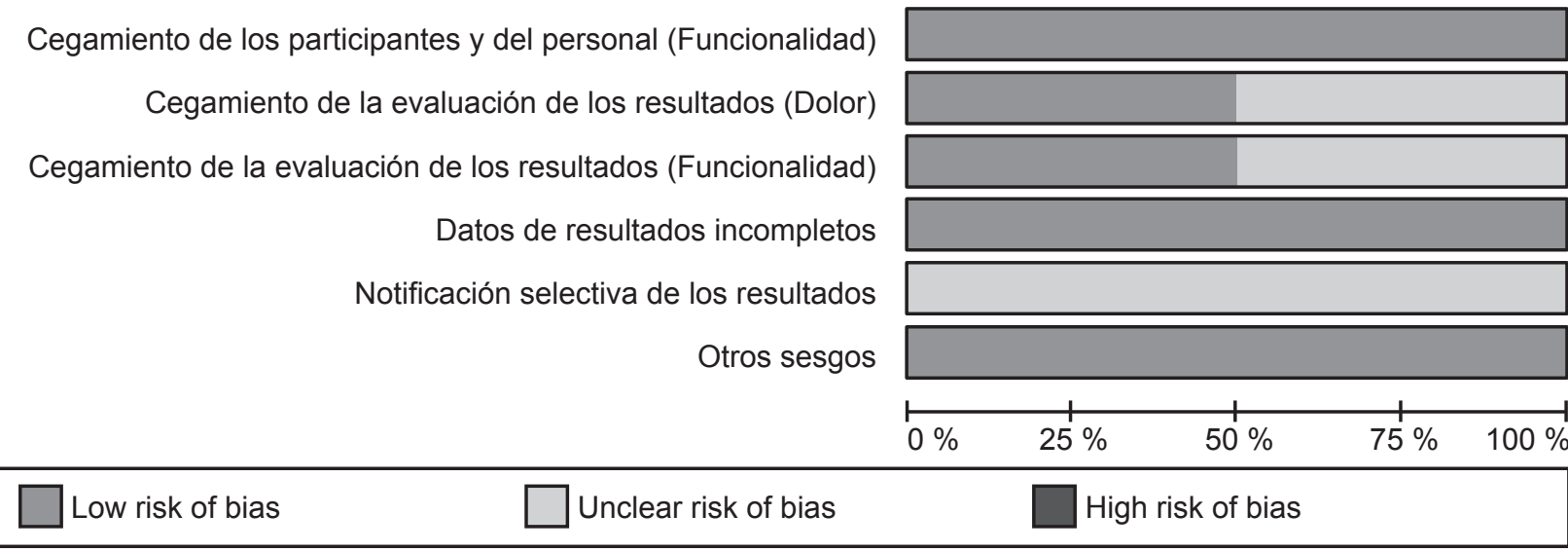

Fig. 2. Resumen del análisis del riesgo de sesgo.

El resumen de todos los aspectos metodológicos de los artículos seleccionados se detalla en la tabla II.

En la tabla III se muestra un resumen de los hallazgos para la comparación taping versus otras intervenciones para la funcionalidad (seguimiento la primera y segunda semana); dolor en resposo (segunda semana); y dolor al movimiento activo (primera y segunda semana).

\section{Descripción de los estudios}

\section{Taping solo versus placebo}

Un estudio ( $\mathrm{n}=42$ pacientes $)$ investigó la efectividad del taping solo comparado con taping placebo en pacientes con pinzamiento sub-acromial (62).

\section{Thelen (2008)}

Se estudió la efectividad del taping comparado con taping placebo en 42 pacientes con pinzamiento sub-acromial. Se aleatorizaron en dos grupos: grupo A (taping) $n=$ 21 pacientes y grupo $\mathrm{B}$ (taping placebo) $\mathrm{n}=21$ pacientes. En el grupo A se realizó la aplicación del taping estándar de hombro para patologías como pinzamiento sub-acromial, propuesta por Kase y cols. (75). La intervención mediante el taping consiste en fijar una cinta en forma de "Y" sobre el deltoides (la aplicación de taping no lleva tensión). Otra cinta de taping va en posición en el trapecio superior simulando la dirección y acción del supraespinoso (sin tensión). La tercera cinta lleva tensión entre unos 50-75 \% y se aplica en forma de "Y" proporcionando una corrección mecánica. En el grupo B se aplicó un taping falso, con dos cintas de $10 \mathrm{~cm}$, una sobre la tuberosidad deltoidea de manera horizontal y otra sobre el trapecio superior de manera vertical, ambas sin tensión. El tratamiento tuvo una duración de 48 a 72 horas. En el análisis de los resultados el grupo de taping placebo no demostró diferencias significativas en ninguna medida de resultado post-aplicación (24 horas) ( $p$ $=0,49)$. Para la medida de resultado de rango de movimiento (ROM) mostró diferencia significativa post-aplicación para el grupo de tratamiento de taping $(\mathrm{p}=0,05)$. Para la medida de resultado de funcionalidad medida con el score SPADI mostró diferencias significativas para ambos grupos en el tercer y sexto día posterior a la aplicación $(\mathrm{p}=0,01)$.

\section{Taping solo versus otros tratamientos}

Un estudio investigó la efectividad del taping solo comparado con otros tratamientos en 55 pacientes con pinzamiento sub-acromial (74).

\section{Kaya (2011)}

Se estudió la efectividad del taping comparado con un programa de terapia física en 55 pacientes con pinzamiento sub-acromial. Se aleatorizaron en dos grupo: grupo A (taping) $n=30$ pacientes y grupo $B$ (terapia física) $n=25$ pacientes. En el grupo A se realizó la aplicación del taping estándar de hombro para patologías como pinzamiento sub-acromial propuesta por Kase y cols. (75). Esta técnica 
TABLA II. CARACTERÍSTICAS DE LOS ESTUDIOS INCLUIDOS

\begin{tabular}{|c|c|c|c|c|}
\hline Autor/año & Condición/Método & $\begin{array}{c}\text { Características } \\
\text { de los pacientes }\end{array}$ & Intervención & $\begin{array}{c}\text { Seguimientos/ } \\
\text { Resultados }\end{array}$ \\
\hline $\begin{array}{l}\text { Thelen et } \\
\text { al., } 2008 \\
(62)\end{array}$ & $\begin{array}{l}\text { SPS: se crearon } \\
\text { los grupos por } \\
\text { un generador } \\
\text { aleatorio: } \\
\text { Grupo A: taping } \\
\text { Grupo B: taping } \\
\text { placebo }\end{array}$ & $\begin{array}{l}\mathrm{n}=42 \\
\text { Grupo A: } \mathrm{n}=21 \text {; } \\
\text { edad: } 18-24 \text { años } \\
\text { Grupo B: } \mathrm{n}=21 \text {; } \\
\text { edad: } 18-24 \text { años }\end{array}$ & $\begin{array}{l}\text { Intervención: } 48 \text { a } 72 \text { horas } \\
\text { Grupo A: aplicación del taping en } \\
\text { forma de Y: } 1^{\circ} \text { sobre el deltoides, } 2^{\circ} \\
\text { cinta en el trapecio superior y la } 3^{\circ} \\
\text { cinta con tensión de } 50-75 \% \text { se aplica } \\
\text { en forma de Y para la corrección } \\
\text { mecánica } \\
\text { Grupo B: aplicación de taping falso, } \\
2 \text { cintas de } 10 \mathrm{~cm} \text {, una cinta sobre la } \\
\text { tuberosidad deltoidea (horizontal) y } \\
\text { la otra cinta sobre el trapecio superior } \\
\text { (vertical) }\end{array}$ & $\begin{array}{l}\text { Seguimiento: } 6 \text { días } \\
\text { Funcionalidad } \\
\text { con el Índice de } \\
\text { Discapacidad } \\
\text { (SPADI) } \\
\text { Rango activo de } \\
\text { movimiento (ROM) } \\
\text { Dolor con Escala } \\
\text { Visual Análoga } \\
\text { (EVA) }\end{array}$ \\
\hline $\begin{array}{l}\text { Kaya et al., } \\
2011(74)\end{array}$ & $\begin{array}{l}\text { SPS: se crearon } 2 \\
\text { grupos aleatorios: } \\
\text { Grupo A: taping } \\
\text { Grupo B: terapia } \\
\text { física }\end{array}$ & $\begin{array}{l}\mathrm{n}=55 \\
\text { Grupo A: } \mathrm{n}=30 ; \\
\text { 18-70 años } \\
\text { Grupo B: } \mathrm{n}=25 ; \\
\text { edad 18-70 años }\end{array}$ & $\begin{array}{l}\text { Intervención: } 3 \text { días } \\
\text { Grupo A: aplicación de taping en } \\
\text { hombro, en forma de Y sobre el } \\
\text { deltoides, trapecio superior y la cinta } \\
\text { en forma de Y con tensión de } 50-70 \% \\
\text { Grupo B: programa de ejercicios } \\
\text { en casa, ejercicios isométricos, } \\
\text { fortalecimiento, elongación } 2 \text { veces } \\
\text { a día y aplicación de agentes físicos } \\
\text { como US a } 1 \mathrm{MHz} \text { a } 1 \mathrm{~W} / \mathrm{cm}^{2} \text { por } \\
5 \text { minutos y TENS y hot packs por } \\
20 \text { minutos al día }\end{array}$ & $\begin{array}{l}\text { Seguimiento: } 2 \\
\text { semanas } \\
\text { Funcionalidad } \\
\text { con Escala de } \\
\text { Discapacidad de } \\
\text { brazo, hombro y } \\
\text { mano (DASH) } \\
\text { Dolor con Escala } \\
\text { Visual Análoga } \\
\text { (EVA) }\end{array}$ \\
\hline
\end{tabular}

también ayuda a mantener la estabilidad escápulo-torácica mediante la corrección mecánica. La aplicación del taping consiste en fijar una cinta sin tensión en forma de "Y" sobre el deltoides. Se aplica otra cinta sin tensión, ubicada en el trapecio superior simulando la dirección y acción del supraespinoso. La tercera cinta va a lo largo de la cinta en forma de "Y" proporcionando una corrección mecánica con una tensión de un 50-75\%. En el grupo B se realizaron modalidades de terapia física que consistía en programa de ejercicios en casa, ejercicios isométricos, fortalecimiento de serrato, trapecio, rotadores externos y elongaciones del pectoral menor. Además se aplicaron agentes físicos como: US a $1 \mathrm{MHZ}$ a $1 \mathrm{~W} / \mathrm{cm}^{2}$ por 5 minutos, hot packs y TENS. El tratamiento constó de 3 posturas de taping en intervalos de 3 días. En el análisis de los resultados de funcionalidad y dolor, ambos grupos muestran mejorías en comparación con la línea de base. En la medida de resultado de dolor el grupo de taping mostró diferencias estadísticamente significativas en comparación con el grupo de terapia física en la primera semana de seguimiento $p=0,001$. Sin embargo no hay diferencias estadísticamente significativas entre ambos grupos en los mismos parámetros en la segunda semana de seguimiento $\mathrm{p}=0,218$.

\section{DISCUSIÓN}

Se intentó determinar el efecto del taping sobre el dolor en sujetos con pellizcamiento sub-acromial. Para lograrlo se realizó una síntesis de la evidencia disponible para responder la interrogante planteada precedentemente: en pacientes con pellizcamiento sub-acromial, ¿el uso del taping solo o en conjunto con otras intervenciones terapéuticas disminuye el dolor?

En un comienzo, debido a la amplia variedad y a la naturaleza de las intervenciones estudiadas, así como también al tipo y tamaño de las poblaciones, medidas de resultado utilizadas, al seguimiento de los pacientes, al informe y al tratamiento estadístico de las variables se hacía bastante compleja la extracción de los datos. Por esto, sólo fueron los dos artículos seleccionados por nuestra búsqueda los que no estaban en condiciones de poder agruparse en una comparación a partir de un estimador puntual, debido a la diversidad de las intervenciones de esta revisión sistemática. Por este motivo entendemos que, para llevar a cabo un meta-análisis se debe evaluar además la homogeneidad estadística, que es menos plausible de realizar debido que no se cumple el primer criterio. 
TABLA III. RESUMEN DE LOS HALLAZGOS PARA LAS COMPARACIONES

\begin{tabular}{|c|c|c|c|c|c|c|}
\hline \multirow{3}{*}{ Resultados } & \multicolumn{2}{|c|}{ Comparación de riesgos ilustrativos } & \multirow{3}{*}{$\begin{array}{c}\text { Efecto } \\
\text { relativo } \\
(I C \text { del } 95 \%)\end{array}$} & \multirow{3}{*}{$\begin{array}{c}N^{\circ} \text { de } \\
\text { participantes } \\
\text { (estudios) }\end{array}$} & \multirow{3}{*}{$\begin{array}{c}\text { Calidad de } \\
\text { la evidencia } \\
\text { (GRADE) }\end{array}$} & \multirow{3}{*}{ Comentarios } \\
\hline & Riesgo asumido & $\begin{array}{c}\text { Riesgo } \\
\text { correspondiente }\end{array}$ & & & & \\
\hline & Control & Taping & & & & \\
\hline $\begin{array}{l}\text { Funcionalidad } \\
\text { DASH }^{1} \text {. } \\
\text { Seguimiento: } \\
\text { mediana } \\
2 \text { semanas }\end{array}$ & $\begin{array}{c}\text { La media } \\
\text { funcionalidad } \\
\text { en los grupos de } \\
\text { control fue } \\
56\end{array}$ & $\begin{array}{l}\text { La media de } \\
\text { funcionalidad } \\
\text { en los grupos de } \\
\text { intervención fue } \\
\text { 57,5 superior }\end{array}$ & - & $\begin{array}{c}55 \\
\text { (1 estudio) }\end{array}$ & $\begin{array}{c}\oplus \oplus \ominus \ominus \\
\text { bajo }^{2}\end{array}$ & - \\
\hline $\begin{array}{l}\text { Funcionalidad } \\
\text { DASH. } \\
\text { Seguimiento: } \\
\text { mediana } \\
1 \text { semana }\end{array}$ & $\begin{array}{c}\text { La media } \\
\text { funcionalidad } \\
\text { en los grupos de } \\
\text { control fue } \\
31\end{array}$ & $\begin{array}{l}\text { La media de } \\
\text { funcionalidad } \\
\text { en los grupos de } \\
\text { intervención fue } \\
18 \text { superior }\end{array}$ & - & $\begin{array}{c}55 \\
\text { (1 estudio) }\end{array}$ & $\begin{array}{c}\oplus \oplus \ominus \ominus \\
\text { bajo }^{3}\end{array}$ & - \\
\hline $\begin{array}{l}\text { Dolor en reposo } \\
\text { EVA. } \\
\text { Seguimiento: } \\
\text { mediana } \\
2 \text { semanas }\end{array}$ & $\begin{array}{l}\text { El dolor medio } \\
\text { en reposo en el } \\
\text { grupo control } \\
\text { fue } 30\end{array}$ & $\begin{array}{l}\text { El dolor medio } \\
\text { en reposo en } \\
\text { los grupos de } \\
\text { intervención fue } \\
\text { O superior }\end{array}$ & - & $\begin{array}{c}55 \\
\text { (1 estudio) }\end{array}$ & $\begin{array}{c}\oplus \oplus \Theta \Theta \\
\text { bajo }\end{array}$ & - \\
\hline $\begin{array}{l}\text { Dolor al } \\
\text { movimiento } \\
\text { activo } \\
\text { EVA. } \\
\text { Seguimiento: } \\
\text { mediana de } \\
1 \text { semana }\end{array}$ & $\begin{array}{l}\text { El dolor medio } \\
\text { al movimiento } \\
\text { activo en los } \\
\text { grupos de } \\
\text { control fue } \\
70\end{array}$ & $\begin{array}{l}\text { El dolor medio } \\
\text { al movimiento } \\
\text { activo en los } \\
\text { grupos de } \\
\text { intervención fue } \\
50 \text { superior }\end{array}$ & - & $\begin{array}{c}55 \\
\text { (1 estudio) }\end{array}$ & $\begin{array}{c}\oplus \oplus \ominus \ominus \\
\text { bajo }\end{array}$ & - \\
\hline $\begin{array}{l}\text { Dolor al } \\
\text { movimiento } \\
\text { activo } \\
\text { EVA. } \\
\text { Seguimiento: } \\
\text { mediana } \\
2 \text { semanas }\end{array}$ & $\begin{array}{l}\text { El dolor medio } \\
\text { al movimiento } \\
\text { activo en los } \\
\text { grupos de } \\
\text { control fue } \\
40\end{array}$ & $\begin{array}{l}\text { El dolor medio } \\
\text { al movimiento } \\
\text { activo en los } \\
\text { grupos de } \\
\text { intervención fue } \\
30 \text { superior }\end{array}$ & - & $\begin{array}{c}55 \\
\text { (1 estudio) }\end{array}$ & $\begin{array}{c}\oplus \oplus \Theta \Theta \\
\text { bajo }\end{array}$ & - \\
\hline $\begin{array}{l}\text { Efectos adversos- } \\
\text { no reportado }\end{array}$ & Ver comentario & Ver comentario & No estimable & - & $\begin{array}{c}\text { Ver } \\
\text { comentario }\end{array}$ & - \\
\hline
\end{tabular}

* La base para el riesgo asumido (por ejemplo, la mediana del riesgo del grupo control en todos los estudios) se suministra en las notas. El riesgo correspondiente (y su intervalo de confianza del $95 \%$ ) se basa en el riesgo asumido en el grupo de comparación y el efecto relativo de la intervención (y su IC $95 \%$ ). IC: intervalo de confianza.

GRADE:

Alta calidad: la investigación adicional es muy poco probable que cambie nuestra confianza en la estimación del efecto. Calidad moderada: la investigación adicional es probable que tenga un impacto importante sobre nuestra confianza en la estimación del efecto y puede cambiar la estimación.

Baja calidad: la investigación adicional es muy probable que tenga un impacto importante sobre nuestra confianza en la estimación del efecto y es probable que cambien la estimación.

Calidad muy baja: no estamos muy seguros sobre la estimación.

${ }^{1}$ La discapacidad de brazo, hombro y mano. ${ }^{2}$ El estudio presentación bajo riesgo de sesgo. ${ }^{3}$ La discapacidad de brazo, hombro y mano. 
De acuerdo a la evaluación del riesgo de sesgo de los artículos incluidos (riesgo de sobreestimar o subestimar los resultados), realizada por los autores de la presente revisión, presenta bajo riesgo de sesgo Thelen (62), moderado riesgo de sesgo Kaya (74). Cabe señalar que el estudio de Thelen fue el único en donde se describía cómo se aleatorizó, sin embargo no se describe cómo se ocultó la aleatorización. En ambos estudios hubo cegamiento de participantes y personal, sin embargo en el estudio de Kaya (74) no se pudo determinar el ocultamiento de los evaluadores de las medidas de resultados. Todos los estudios seleccionados contemplaron la exclusión de los pacientes o el manejo adecuado por análisis de intención de tratar. Ninguno de los estudios tiene inscrito el protocolo de investigación en algún sistema de registro existente en la actualidad. Según esto se hace muy difícil poder establecer si hubo un reporte selectivo adecuado de medidas de resultados. En la actualidad existe una RS del tema propuesto previamente, Copping 2005 (71), en donde determina la efectividad del tape en la propiocepción y reclutamiento muscular en pacientes con pinzamiento sub-acromial. No obstante no reporta resultados concluyentes sobre la intervención estudiada y propone nuevas investigaciones para determinar el efecto del taping. Por ende se hace plausible realizar esta revisión sistemática con una metodología más acabada. Sin perjuicio de todo lo anteriormente expuesto, la falta de agrupación de los datos hace más difícil establecer las opciones terapéuticas con mayor efectividad para esta condición clínica. Por esto, todas las consideraciones mencionadas anteriormente deben ser evaluadas cuando se interpreten los resultados de los diferentes estudios y se intenten establecer conclusiones acerca de la efectividad clínica del taping en este tipo de pacientes.

\section{CONCLUSIÓN}

Evidencia de baja calidad sugiere que el taping sería efectivo en mejorar la funcionalidad del hombro hasta en una semana posterior a su aplicación en sujetos que presentan pellizcamiento sub-acromial.

Evidencia de baja calidad sugiere que la aplicación de taping sería efectivo en reducir el dolor en reposo y durante los movimientos activos en sujetos que presentan pellizcamiento sub-acromial, hasta una semana posterior de su aplicación en comparación con un programa de terapia física.

$\mathrm{Al}$ momento de aplicar los resultados de la presente revisión sistemática se debe considerar que la calidad global de la evidencia de todos los resultados de interés en nuestra revisión sistemática es clasificada como "baja", esto quiere decir que es muy probable que investigaciones adicionales tengan un impacto importante en la confianza de la estimación del efecto y es probable que cambie. Por tal motivo encontramos que es razonable la utilización de taping para el manejo del dolor y funcionalidad a corto plazo (1 semana) en sujetos que presenten pellizcamiento sub-acromial. También debemos considerar que la decisión para utilizar el taping debe basarse en los costos, las preferencias del paciente y la seguridad de la opción de tratamiento.

CORRESPONDENCIA:

R. A. Aguilera Eguía

e-mail:kine.rae@gmail.com

\section{BIBLIOGRAFÍA}

1. Lo YP, Hsu YC, Chan KM. Epidemiology of shoulder impingement in upper arm sports events. Br J Sports Med 1990;24:173-7.

2. Bot S, van der Waal J, Terwee C, van der Windt D, Schellevis F, Bouter L, Dekker J. Incidence and prevalence of complaints of the neck and upper extremity in general practice. Ann Rheum Dis 2005;64:118-23.

3. Picavet H, Schouten J. Musculoskeletal pain in the Netherlands: Prevalences, consequences and risk groups, the DMC(3)-study. Pain 2003;102(1-2):167-78.

4. Michener L, McClure P, Karduna A. Anatomical and biomechanical mechanisms of sub-acromial impingement syndrome. Clin Biomech 2003;18(5):369-79.

5. Green S, Buchbinder R, Hetrick S. Physiotherapy interventions for shoulder pain. Cochrane Database Syst Rev 2003;CD004258. Available at: http://dx.doi. org/10.1002/14651858.CD004258.

6. Johansson KM, Adolfsson LE, Foldevi MO. Effects of acupuncture versus ultrasound in patients with impingement syndrome: Randomized clinical trial. Phys Ther 2005;85:490-501.

7. Imhoff A, Ledermann T. Definition, pathologische Befunde und Pathogenese. In: Eulert J, Hedtmann A, editors. Das Impingementsyndrom der Schulter. Stuttgart: Thieme; 1996. p. 1-13.

8. Neer C. Impingement lesions. Clin Orthop 1983;173:70-7.

9. Neer $C, 2^{\text {nd }}$. Anterior acromioplasty for the chronic impingement syndrome in the shoulder: A preliminary report. J Bone Joint Surg Am 1972;54:41-50.

10. Hébert LJ, Moffet H, Dufour M, Moisan C. Acromiohumeral distance in a seated position in persons with impingement syndrome. J Magn Reson Imaging 2003;18:72-9.

11. Graichen H, Bonel H, Stammberger T, Haubner M, Rohrer $\mathrm{H}$, Englmeier KH, et al. Three-dimensional analysis of the width of the sub-acromial space in healthy subjects and patients with impingement syndrome. AJR Am J Roentgenol 1999;172:1081-6.

12. Roy JS, Moffet H, Hébert LJ, Lirette R. Effect of motor control and strengthening exercises on shoulder function in persons with impingement syndrome: A single-subject study design. Man Ther 2009;14:180-8.

13. Morrison M, Greenbaum M, Einhorn. Shoulder impingement conservative management of shoulder injuries. Orthopedic Clinics of North America 2000;31(2):285-93.

14. Matsen F. Sub-acromial impingement. In: Rockwood C, Matsen A. The shoulder. Philadelphia: Saunders; 1990. p. 623-46.

15. Kase K, Wallis J. Clinical therapeutic applications of the kinesio taping method. Axon; 2003.

16. Moffe H, Hebert L, Dufour L, Tardif J. Variation in sub-acromial distance measured by magnetic resonance imaging 
during shoulder flexion and abduction movements. Can J Rehabil 1998;11:265-67.

17. Bartolozzi A, Andreychik D, Ahmad S. Determinants of outcome in the treatment of rotator cuff disease. Clin Orthop 1994;308:90-7.

18. Bigliani LU, Morrison DS, April EW. The morphology of the acromion and its relationship to rotator cuff tears. Orthop Trans 1986;10:228.

19. Morrison DS, Frogameni AD, Woodworth P. Non-operative treatment of sub-acromial impingement syndrome. J Bone Joint Surg Am 1997;79:732-7.

20. Peters G, Kohn D. Mid-term clinical results after surgical versus conservative treatment of sub-acromial impingement syndrome [in German]. Unfallchirurg 1997;8:623-9.

21. Townsend H, Jobe FW, Pink M, Perry J. Electromyographic analysis of the glenohumeral muscles during a baseball rehabilitation program. Am J Sports Med 1991;19:264-72.

22. Toivonen DA, Tuite MJ, Orwin JF. Acromial structure and tears of the rotator cuff. J Shoulder Elbow Surg 1995;4:37683.

23. Morrison DS, Bigliani LU. The clinical significance of variations in acromial morphology. Orthop Trans 1987;11:234.

24. Chen SK, Simonian PT, Wickiewicz TL. Radiographic evaluation of glenohumeral kinematics. A muscle fatigue model. J Shoulder Elbow Surg 1999;8:49-52.

25. Lewis JS, Green AS, Dekel S. The aetiology of sub-acromial impingement syndrome. Physiotherapy 2001;87(9):458-69.

26. Ludewig P, Cook T. Alterations in shoulder kinematics and associated muscle activity in people with symptoms of shoulder impingement. Phys Ther 2000;80(3):276-91.

27. Kamkar A, Irrgang J, Whitney S. Nonoperative management of secondary shoulder impingement syndrome. J Orthop Sports Phys Ther 1997;17:212-24.

28. Kibler W. Scapular involvement in impingement: Signs and symptoms. Instr Course Lect 2006;55:35-43.

29. Borstad J, Ludewig P. Comparison of scapular kinematics between elevation and lowering of the arm in the scapular plane. Clin Biomech (Bristol, Avon) 2002;17:650-9.

30. McClure P, Michener L, Karduna A. Shoulder function and 3 dimensional scapular kinematics in people with and without shoulder impingement syndrome. Phys Ther 2006;86:1075-90.

31. Bigliani L, Levine W. Sub-acromial impingement syndrome. J Bone Joint Surg Am 1997;79:1854-68.

32. Steenbrink F, de Groot J, Veeger H, Meskers C, van de Sande M, Rozing P. Pathological muscle activation patterns in patients with massive rotator cuff tears, with and without sub-acromial anaesthetics. Man Ther 2006;11:231-7.

33. Lewis JS, Wright C, Green A. Sub-acromial impingement syndrome: The effect of changing posture on shoulder range of movement. Journal of Orthopaedic Sports Physical Therapy $2005 ; 35(2): 72-87$.

34. Halseth T, McChesney JW, DeBeliso M, Vaughn R, Lien J. The effects of KinesioTM taping on proprioception at the ankle. J Sports Sci Med 2004;3:1-7.

35. Kase K, Tatsuyuki H, Tomoki O. Development of Kinesio ${ }^{\mathrm{TM}}$ tape. In: Kinesio Taping ${ }^{\mathrm{TM}}$ Perfect Manual. Kinesio Taping Association; 1996:6-10, 117-8.

36. Kase K. Illustrated kinesio taping. $2^{\text {nd }}$ ed. Tokyo: Ken'ikai Information; 1994. p. 6-9:73.

37. Merino R. Efecto del kinesio taping en el rango de movimiento de la cadera y zona lumbar en triatletas. Un estudio piloto. J Sport Health 2010;2:109-18.

38. Miller P, Phty B, Cert GH, Osmomotherly P. Does scapula taping facilitate recovery for shoulder impingement symp- toms? A pilot randomized controlled trial. The Journal of Manual \& Manipulative Therapy 2009;17(1):E6- 13.

39. Chen PL, Hong WH, Lin CH, Chen WC. Biomechanics effects of kinesio taping for persons with patellofemoral pain syndrome during stair climbing. In: Abu Osman NA, Ibrahim F, Wan Abas WAB, Abd Rahman HS, Ting HN, editors. Biomed 2008, Proceedings 21. Berlin: Springer; 2008. p. 395-7.

40. Goo J. A new step for treatment of ankle sprain [in Japanese]. 16th Annual Kinesio taping International Symposium Review 2001;16-9.

41. Oliveria R. Soft tissue injuries in sports people - The contribution of kinesio taping [in Japanese]. $15^{\text {th }}$ Annual Kinesio taping International Symposium Review 1999;13-23.

42. Yoshida A, Kahanov L. The effect of kinesio taping on lower trunk range of motions. Res Sports Me 2007;15:103-12.

43. Host HH. Scapular taping in the treatment of anterior shoulder impingement. Physical Therapy 1995;75:803-12.

44. Morrissey D. Proprioceptive shoulder taping. Journal of Bodywork and Movement Therapies 2000;4:189-94.

45. Yasukawa A, Patel P, Sisung C. Pilot study: Investigating the effects of Kinesio Taping ${ }^{\mathbb{B}}$ in an acute pediatric rehabilitation setting. American Journal of Occupational Therapy 2006;60:104-10.

46. Macdonald R. Taping Techniques: Principles and Practice. Oxford, UK: Butterworth-Heinemann Ltd; 1994.

47. Frazier S, Whitman J, Smith M. Utilization of kinesio tex tape in patients with shoulder pain or dysfunction: A case series. Advanced Healing 2006:8-20.

48. Jaraczewska E, Long C. Kinesio taping in stroke: Improving functional use of the upper extremity in hemiplegia. Top Stroke Rehabil. 2006;13:31-42. Available at: http://dx.doi. org/10.1310/33KAXYE3-QWJB-WGT6.

49. Murray H, Husk LJ. Effect of kinesio taping on proprioception in the ankle [abstract]. J Orthop Sports Phys Ther. 2001;31:A37.

50. Osterhues DJ. The use of Kinesio Taping in the management of traumatic patella dislocation. A case study. Physiother Theor Pract 2004;20:267-70.

51. García F, Rodríguez A, Herrero A. Treatment of myofascial pain in the shoulder with kinesio taping. A case report. Man Ther 2010;15:292-5.

52. Tsai C, Chang W, Lee J. Effects of short-term treatment with kinesiotaping for plantar fasciitis. J Musculoskeletal Pain 2010;18:71-80.

53. Pérez P, Gascó J, Merino M, Sandá A, Moll R, Castillo V. Influence of the neuromuscular bandage on plantar pressure during walking. Fisioterapia 2010;32:111-5.

54. Karlsson J, Andreasson GO. The effect of external ankle support in chronic lateral ankle joint instability: An electromyographic study. The American Journal of Sports Medicine 1992;20(3):257-61.

55. Cools AM, Witvrouw EE, Danneels LA, Cambier DC. Does taping influence electromyographic muscle activity in the scapular rotators in healthy shoulders? Man Ther 2002; 7:154-62

56. You SH, Granata KP, Bunker LK. Effects of circumferential ankle pressure on ankle proprioception, stiffness, and postural stability: a preliminary investigation. J Orthop Sports Phys Ther 2004;34:449-60.

57. Słupik A, Dwornik M, Bialoszewski D, Zych E. Effect of kinesio taping on bioelectrical activity of vastus medialis muscle. Preliminary report. Ortop Traumatol Rehabil 2007;9:644-51 
58. Simoneau G, Degner R, Kramper C, Kittleson K. Changes in ankle joint proprioception resulting from strips of athletic tape applied over skin. J Athl Train 1997;32:141-7.

59. Sijmonsma J. Manual de taping neuro muscular. Portugal; 2007 Aneid press.

60. González J, Fernández C, Cleland J, Huijbregts P, Del Rosario Gutiérrez M. Short-term effects of cervical kinesio taping on pain and cervical range of motion in patients with acute whiplash injury: A randomized clinical trial. J Orthop Sports Phys Ther 2009;39:515-21.

61. OEliwiñski Z, Senderek T. Kinezjotaping 2 nowa metoda leczenia? Rehabilitacja W Praktyce 2007;3:18-20.

62. Thelen M, Dauber J, Stoneman P. The clinical efficacy of kinesio tape for shoulder pain: A randomized, double-blinded, clinical trial. J Orthop Sports Phys Ther 2008;38:389-95.

63. Hsu Y, CheN W, Hsiu-Chen L, Wendy T, Yi-Fen S. The effects of taping on scapular kinematics and muscle performance in baseball players with shoulder impingement syndrome. Journal of Electromyography and Kinesiology 2009;13. [Epub ahead of print].

64. McConnell J. The management of chondromalacia patellae: A long-term solution. Aust J Physiother 1986;23:215-23.

65. Birrer RB, Poole B. Athletic taping, part 4: the shoulder and elbow: added support enables the athlete to remain active. J Musculoskel Med 1996;1:52-7.

66. Sundaramurthy N, Nehru A, Rajalakshmi D. Effect of taping as a component of conservative treatment for sub-acromial impingement syndrome. Health 2012;4(4):237-41.

67. Kalter J, Apeldoorn A, Ostelo R, Henschke N, Knol D, Van Tulder N. Taping patients with clinical signs of sub-acromial impingement syndrome: The design of a randomized controlled trial. BMC Musculoskeletal Disorders 2011;12:188.

68. Greene TA, Wight CR. A comparative support evaluation of three ankle orthoses before, during and after exercise. Journal of Orthopedic and Sports Physical Therapy 1990;11:453-66.

69. Gross MT, Batten AL, Lamm AL. Comparison of Donjoy ankle ligament protector and subtalar sling ankle taping in restricting foot and ankle motion before and after exercise. Journal of Orthopedic and Sports Physical Therapy 1994;19(1):33-41.

70. Lohrer H, Alt W, Gollhofer A. Neuromuscular properties and functional aspects of taped ankles. The American Journal of Sports Medicine 1999;27(1):69-75.

71. Copping J, O'driscoll M. Application of tape at the shoulder joint: An effective therapeutic modality for the treatment of impingement syndrome? Physical Therapy Reviews 2005; 10:231-6.

72. Dickersin K, Scherer R, Lefebvre C. Identifying relevant studies for systematic reviews. BMJ 1994;309:1286-91.

73. Higgins JPT, Green S, editors. Cochrane Handbook for Systematic Reviews of Interventions. Version 5.0.1 [Updated September 2008]. The Cochrane Collaboration 2008. Available at: www.cochrane-handbook.org.

74. Kaya E, Zinnuroglu M, Tugcu I. Kinesio taping compared to physical therapy modalities for the treatment of shoulder impigement syndrome. Clin Rheumatol 2010 doi:10.1007/ s10067-010-1475-6.

75. Kase K, Wallis J, Kase T. Clinical therapeutic applications of the kinesiotaping method. Tokyo, Japan: KenIkai Co Ltd; 2003. 